\title{
HARALd Burger
}

\section{Phraseologie - Kräuter und Rüben? \\ Traditionen und Perspektiven der Forschung}

\begin{abstract}
Den Nagel auf den Kopf treffen und einen Nagel einschlagen - mit diesen beiden Ausdrücken wird das Spektrum der phraseologischen Wortverbindungen zwischen stärkerer und schwächerer Festigkeit angezeigt. Damit sind auch zwei hauptsächliche Fragestellungen indiziert, die die Phraseologieforschung seit ihren Anfängen bestimmt haben: einerseits diejenige nach den „,auffälligen“ festen Wortverbindungen, den Idiomen, andererseits diejenige nach den scheinbar selbstverständlichen Verbindungen von Wörtern, die gerade nicht auf den ersten Blick ins Auge fallen, den Kollokationen.

Anschließend an eine Abgrenzung des Objektbereichs der Phraseologie soll eine Skizze einiger Forschungslinien zeigen, welche Bereiche der Phraseologie gut erforscht sind und wo Desiderate bestehen. Etwas genauer wird auf die problematische Schnittstelle von Grammatik und Phraseologie eingegangen. Aus dem Überblick soll einerseits hervorgehen, dass sich Phraseologie, metaphorisch gesprochen, nicht mit Kraut und Rüben befasst, dass aber andererseits eine forschungsgeschichtliche Verschiebung von den bisher als Kräutern gehandelten Idiomen zu den weniger beachteten Rüben zu prognostizieren ist.
\end{abstract}

\section{Konzepte der Phraseologieforschung}

In Gottfried Kellers „Romeo und Julia auf dem Dorfe“ geht es zunächst um zwei Bauern, die einst befreundet waren, sich nun aber wegen eines Ackerstücks, auf das sie gemeinsam Anspruch erheben, in tödliche Feindschaft hineingesteigert haben. Und da heißt es von eben diesem Ackerstück:

„Es war aber keine Rede mehr von einer ordentlichen Bebauung, und auf dem Acker, der einst so schön im gleichmäßigen Korne gewogt, wenn die Ernte kam, waren jetzt allerhand abfällige Samenreste gesäet und aufgegangen, aus alten Schachteln und zerrissenen Düten zusammengekehrt, Rüben, Kraut und dergleichen und etwas Kartoffeln, so daß der Acker aussah wie ein recht übel gepflegter Gemüseplatz und eine wunderliche Musterkarte war, dazu angelegt, um von der Hand in den Mund zu leben, hier eine Handvoll Rüben auszureißen, wenn man Hunger hatte oder nichts Besseres wußte, dort eine Tracht Kartoffeln oder Kraut, und das übrige fortwuchern oder verfaulen zu lassen, wie es mochte."

(Gottfried Keller, Romeo und Julia auf dem Dorfe, Reclam S. 36)

Wenn wir heute diese Passage lesen, wird vielen von uns bei der Stelle Rüben, Kraut ... die Wortverbindung Kraut und Rüben in den Sinn kommen. 
Mit dieser „Paarformel“ meinen wir heutzutage ,unordentlich, durcheinander', im Text von Keller jedoch kann dies nicht in erster Linie gemeint sein, da ja noch andere Konkreta genannt werden, die mit Gemüse zu tun haben (Gemüseplatz, Kartoffeln, eine Handvoll Rüben), ebenso Verben wie fortwuchern, verfaulen, die auf das konkret Gemeinte hindeuten. Die Verbindung als ganze bedeutet heute etwas anderes, als aus der Kombination der Teile in ihrem wörtlichen Verständnis hervorgeht. Sie hat eine „,idiomatische“ Bedeutung.

In dem folgenden Zeitungstext von heute ist ganz unverkennbar, dass auf diese idiomatische Bedeutung angespielt wird:

\section{Bunt wie auf dem Gemüsemarkt}

Der Lehrpfad im grössten Gemüse-Anbaugebiet der Schweiz bietet mehr als Geschichten über Kraut und Rüben. [...]

(Tages-Anzeiger, Zürich, 5.6.98)

Das soll wohl heißen: Der Lehrpfad bietet nicht, Geschichten über alles Mögliche durcheinander', sondern faktische Informationen über die verschiedenen Gemüsesorten. Im Text selber ist dann die Rede von den Realia, von Rotkraut und Zuckerrübenfeld.

In Duden GW findet man unter „Kraut“ folgenden Eintrag:

'Kraut, das; -[e]s, Kräuter [...] 1. Pflanze, deren oberirdische Teile nicht verholzen. 2. Heilpflanze, Würzpflanze o. Ä. [...] 3. <o.Pl.> alles Grüne, Stängel u. Blätter (bes. bei bestimmten Nutzpflanzen im Unterschied zu dem für die menschliche Ernährung verwertbaren Teil): das K. der Rüben, Kartoffeln; die Pflanze wächst, schießt [zu sehr] ins K. (treibt zu viele große Blätter u. lässt deshalb keine gute Blüte bzw. reiche Frucht erwarten); *ins K. schießen (in unliebsamer Weise rasch zunehmen, sich verbreiten; überhand nehmen [...]; wie $K$. und Rüben (ugs.; unordentlich; viell. nach dem Bild eines Rübenackers, auf dem nach der Ernte die abgeschnittenen Blätter der Pflanze u. die Rüben durcheinander liegen od. bezogen auf ein Eintopfgericht, bei dem Kraut [= Kohl] u. Rüben zusammen gekocht werden): es lag alles wie K. und Rüben herum, durcheinander; in seinem Aufsatz geht alles wie K. und Rüben durcheinander; Schein und Sein mengen sich wie K. und Rüben (Spiegel 13, 1981, 214). 4. <o. Pl.> (bes. südd., österr.) [Weiß]kohl: K. anbauen, schneiden, einlegen $[\ldots]$

Der Ausdruck wie Kraut und Rüben ist durch Fettdruck und Sternchen als Redensart gekennzeichnet. Er ist unter Punkt 3 eingeordnet und mit der Bedeutungsangabe ,unordentlich ' versehen, vermutlich weil man annimmt, dass die Komponente Kraut im Phraseologismus semantisch mit diesem Bedeutungspunkt zusammenhängt. Zumindest geht das aus der etymologischen Erläuterung hervor (,vielleicht nach ..."). Wenn allerdings die zweite angebotene Variante für die Etymologie (,oder bezogen auf ein Eintopfgericht ...") zutrifft, würde die Zuordnung nicht mehr stimmen. Dann würde der Ausdruck zu Punkt 4 (,Kohl') gehören. 
Nun würde man noch gerne wissen, ob es sich auch bei Keller schon um eine Anspielung handelt. Das DW gibt unter „Kraut" klare Auskunft (Bd. 5, S. 2109) darüber, dass das Idiom schon alt ist und in der heutigen Bedeutung mindestens bis ins 17. Jahrhundert zurückreicht. Man muss also annehmen, dass der Text Kellers vordergründig ganz auf der wörtlich-konkreten Ebene bleibt, dass hintergründig aber durch den Anklang an das Idiom auch der idiomatische Sinn mitgemeint ist.

Wenn man nun den Ausdruck ein bisschen verändert, z. B. Rüben und Kraut, dann geht die idiomatische Bedeutung wieder verloren. Vollends bei der Verwendung von Kraut im Plural - Kräuter. Gemäß Duden GW kann Kräuter nicht dem Bedeutungspunkt 3 zugeordnet werden, schon deshalb weil Kraut in der Bedeutung 3 keinen Plural hat. Es bleibt nur der Bedeutungspunkt 2 (,Heilpflanze, Würzpflanze'), bei dem der Singular vermutlich seltener ist als der Plural. Man kann aber Kräuter und Rüben in geeignetem Kontext jederzeit auch als eine neue Metapher einsetzen. Kräuter sind sozusagen etwas Feineres als Kraut und auch feiner als Rüben, und bei Kräuter und Rüben würde man sich vielleicht eine Mischung von Feinerem und Banalerem vorstellen.

Phraseologie befasst sich mit den „festen Wortverbindungen“. Dabei heißt „fest" ganz Verschiedenes, z. B. bei Kraut und Rüben: Der Ausdruck ist formal insofern fest, als man die Nomina nicht umstellen kann (Rüben und Kraut würde die idiomatische Bedeutung zerstören). Lexikalisch ist er fest, insofern man die Komponenten nicht austauschen kann (z.B. „Kraut und Möhren"). Auch in semantischer Hinsicht ist er fest, nämlich in dem Sinne, dass die idiomatische Bedeutung für den Ausdruck als Ganzen gilt.

Andere Ausdrücke sind auch idiomatisch, aber die Festigkeitsmerkmale sind anders verteilt, wie bei den Nagel auf den Kopf treffen. Der Ausdruck findet sich in Duden GW als Redensart gekennzeichnet unter "Nagel“, Punkt 1 (,am unteren Ende zugespitzter, am oberen Ende abgeplatteter od. abgerundeter [Metall]stift, der in etw. hineingetrieben wird (u. zum Befestigen von etw. od. zum Verbinden bes. von Holzteilen dient') mit der Bedeutungsangabe ,den Kernpunkt von etw. treffen, erfassen'. In gewissem Maße ist bei dieser Wortverbindung die Stellung der Glieder veränderbar. Das Verb kann wie ein normales Verb in verschiedenen Personen, Tempora, Modi auftreten (z. B. sie haben den Nagel auf den Kopf getroffen).

Festigkeit ist offensichtlich ein graduelles Konzept, und dies unter jedem der betrachteten Aspekte. Am einen Ende der Skala stehen die in vieler Hinsicht verfestigten Ausdrücke, am anderen Ende diejenigen, die nur schwache Merkmale von Festigkeit aufweisen und die man als „Kollokationen“ bezeichnet. 
Dazu gehört zum Beispiel die Wortverbindung einen Nagel einschlagen, die viel weniger fest ist als den Nagel auf den Kopf treffen. In Duden GW finden wir den Ausdruck unter „Nagel“" nur als Beispiel.

Wenn man im Deutschen sagen will, was man mit einem Nagel normalerweise tut, dann sagt man einschlagen. Gewiss kann man - je nach der konkreten Tätigkeit - auch andere Verben wählen (z. B. einhämmern oder hineintreiben), aber das Üblichste ist wohl einschlagen. Das fällt dem Muttersprachler nicht auf, wohl aber demjenigen, der Deutsch als Fremdsprache lernt. Einen Nagel einschlagen hat nur ein Festigkeitsmerkmal: Nagel kommt bevorzugt mit dem Verb einschlagen vor. Im übrigen kann man mit den Komponenten der Wortverbindung alles machen, was die Grammatik erlaubt.

Wie fein die Abstufungen der Festigkeit sein können, sieht man, wenn man einen Nagel einschlagen mit den Tisch decken vergleicht. Auf den ersten Blick sind die beiden Fälle gänzlich parallel. Doch die Wörterbucheinträge zeigen ein leicht anderes Bild:

Unter „Tisch“, Punkt 1. a) (Möbelstück, das aus einer waagerecht auf einer Stütze, in der Regel auf vier Beinen, ruhenden Platte besteht, an der gegessen, gearbeitet, auf die etw. gestellt, gelegt werden kann) findet man in Duden GW unter den Beispielen: ,ein gedeckter T.; der T. war reich gedeckt (es gab reichlich $u$. gut zu essen); [...] den T. ausziehen, decken, abdecken, abwischen [...]" Das Verb decken findet sich hier in einer Reihe mit ausziehen, abdecken, abwischen. Unter decken, Punkt 1 c), steht: „(einen Tisch) zum Essen herrichten, mit einem Tischtuch, Geschirr, Bestecken u.a. versehen: er deckte den Frühstückstisch; es ist für fünf Personen gedeckt; ein liebevoll gedeckter Tisch".

Wenn man die Handlung bezeichnen will, die unter c) paraphrasiert ist, dann sagt man im Deutschen üblicherweise decken (und nicht herrichten oder dergl.). Insoweit stimmt der Befund mit dem bei einen Nagel einschlagen überein. Man sieht aber noch etwas Weiteres: Der semantische Kommentar zu der Tisch war reich gedeckt (,es gab reichlich und gut zu essen') unter „Tisch“ deutet darauf hin, dass es beim Tisch nicht in erster Linie um das ,Möbelstück' geht, sondern dass hinsichtlich der Funktion bereits eine leichte Spezialisierung vom ,Möbelstück' zu einem ,Ort des Essens' stattfindet. Die lexikalisch verfestigte Kombination mit decken führt also zu einer leicht idiomatisierten Gesamtbedeutung. Dies im Gegensatz zu der Kombination mit den Verben ausziehen, abwischen. Im übrigen ist der Ausdruck unauffällig, in grammatischer Hinsicht kann man alles mit ihm machen, was für eine entsprechende nicht phraseologische Konstruktion erlaubt ist.

Kollokationen wie den Nagel einschlagen oder den Tisch decken hält man als Muttersprachler für sozusagen selbstverständlich. Dass sie nicht selbstverständlich sind, sieht man leicht, wenn man die Perspektive des Fremd- 
sprachenlerners einnimmt und z.B. das Deutsche und das Englische vergleicht. Im Großwörterbuch Englisch findet man, um nur die Verbindung den Tisch decken zu nehmen, weder unter „Tisch" noch unter "table“ Aufschlüsse zu dem Problem (ein lexikographisch problematisches Verfahren). Wohl aber gibt es unter den folgenden Verben klare Hinweise auf die Festigkeit der Verbindung:

"decken": $\quad$ den Tisch decken - lay or set the table

"lay": $\quad$ lay the table - den Tisch decken

"set": $\quad$ (lay for meal) - decken (Tisch)

(Duden Oxford)

Welche Aspekte die Festigkeit auf den verschiedenen Ebenen der Sprache hat bzw. haben kann, ist in der Phraseologieforschung ausführlich diskutiert worden (für eine Übersicht vgl. Burger 2003, S. 16 ff.), und insofern ist das Forschungsgebiet der Phraseologie als das Gebiet der ,festen Wortverbindungen" relativ klar abgesteckt.

\section{Forschungstraditionen und ausgewählte Forschungsbereiche}

Es ist unbestritten, dass der Schweizer Charles Bally (vgl. Bally 1909) als einer der Begründer der linguistischen Disziplin Phraseologie gilt. Im Anschluss an Bally hat vor allem die sowjetische Phraseologieforschung (vgl. Jaksche et al. [Hg.] 1981) seit den 40er Jahren die Grundbegriffe der Phraseologie erarbeitet (vor allem die drei Begriffe der "Mehrgliedrigkeit", der „Bedeutungstransformation“ - mit verschiedenen synonymen Termini - und der „Festigkeit“). Diese Forschungen gingen ein in die beiden zusammenfassenden Darstellungen von 1982, diejenige von Fleischer und das Handbuch von Burger/Buhofer/Sialm.

Die Mannheimer Tagung „Phraseologie und Wortbildung“ (1988) ${ }^{1}$ hatte das Ziel, Phraseologie als ein relativ neues Gebiet der Linguistik darzustellen und es gegenüber der bereits etablierten Wortbildungslehre abzugrenzen bzw. Gemeinsamkeiten herauszuarbeiten. So ging es Fleischer in seinem programmatischen Text (1997 [1988]) darum, den Ort der Phraseologie im sprachlichen System zu bestimmen, insbesondere die „Arbeitsteilung“ zwischen Wortbildung und Phraseologie in struktureller und semantischer Hinsicht.

Der Titel der Mannheimer Tagung 2003 ist wesentlich vager, von „mehr oder weniger" ist die Rede. Dies ist ein Symptom für eine Neuorientierung der Forschung, die im folgenden zu erläutern sein wird. Seit damals sind die Literatur zur Phraseologie und das Interesse am Gegenstand sprunghaft angewachsen. Ein Handbuch „Phraseologie“ in der Reihe „Handbücher zur

\footnotetext{
Vgl. den erst 1997 erschienenen Tagungsband Wimmer/Berens (Hg.).
} 
Sprach- und Kommunikationswissenschaft" (HSK) befindet sich in Arbeit ${ }^{2}$, und 1999 wurde die „Europäische Gesellschaft für Phraseologie“ gegründet ${ }^{3}$.

Schon Bally hat zwei wesentliche - wahrscheinlich die wesentlichen - Perspektiven formuliert, die zur Wahrnehmung phraseologischer Phänomene führen: zum einen den affektiven Charakter der Sprache, und zwar primär der Muttersprache, zu untersuchen, zum anderen die Muttersprache aus der Optik der Fremdsprachenlernenden zu sehen. In der Phraseologie sind es zwei unterschiedliche Aspekte, die dadurch in den Blick kommen: Die Idiome von der Art Kraut und Rüben oder den Nagel auf den Kopf treffen sind interessant wegen ihrer Expressivität, ihres ,affektiven Charakters" in der Terminologie von Bally, die Ausdrücke mit geringer Festigkeit wie den Tisch decken, weil sie Bindungen aufweisen, die für die Lernenden unerwartet sind. Vom Standpunkt des Muttersprachlers sind sie gänzlich unauffällig, man bemerkt sie erst im Sprachkontrast oder im Kontrast zwischen verschiedenen historischen Stufen der gleichen Sprache. Diese Doppelheit der Perspektiven ist bis heute erhalten geblieben.

Ich möchte nun exemplarisch drei Aspekte der neueren Forschung herausgreifen, die sich in einem unterschiedlichen Stadium der Bearbeitung befinden. Beim ersten handelt es sich um einen der bereits gut erforschten Bereiche der Phraseologie, beim zweiten um einen in Diskussion befindlichen Fragenkreis, der dritte Aspekt ist der problematischste, weshalb ich ihn etwas ausführlicher bespreche. Ich kann jeweils nur wenige Literaturhinweise geben und verweise im übrigen auf die im Internet verfügbaren Bibliographien von Europhras und Euralex (s.u. unter Literatur).

\subsection{Phraseologie im Text}

Untersucht wurde in den letzten zwanzig Jahren auf breiter Basis, wie und in welchen Funktionen Phraseologismen in Texten und Situationen, insbesondere im heutigen Deutsch vorkommen. ${ }^{4}$ Hier standen und stehen die Idiome im Vordergrund, also insbesondere die auffälligen Vorkommen fester Wortverbindungen.

Es gibt eine Reihe deutlicher Indizien dafür, dass Idiome wegen ihres auffälligen Charakters in Texten gebraucht werden. Ich nenne einige, die mir wichtig erscheinen:

${ }^{2}$ Hg. von Harald Burger, Dmitrij Dobrovol'skij, Peter Kühn, Neal R. Norrick. Mouton de Gruyter. Berlin.

${ }^{3}$ Es ist inzwischen gelungen, verschiedenste Richtungen der Phraseologieforschung und auch verwandte Richtungen auf mehreren großen und kleineren Tagungen zusammenzuführen, vgl. die Tagungsbände von Europhras (s. www.europhras.unizh.ch).

4 Vgl. z. B. die Tagungsbände von Europhras (s. die Angaben unter www.europhras. unizh.ch) bzw. die Arbeiten in der Reihe „Phraseologie und Parömiologie“, hg. von Wolfgang Eismann, Peter Grzybek, Wolfgang Mieder. 
(1) Idiome kommen bevorzugt an bestimmten Orten im Text vor, damit verknüpft: sie haben dort bevorzugte Funktionen. In manchen Arten von Medientexten z. B. bilden sie oft den Rahmen des Textes - d.h. ein und dasselbe Idiom bzw. semantisch verwandete Idiome stehen in der Schlagzeile und werden am Ende des Textes wieder aufgenommen. Z. B. erweckt die Schlagzeile eine - vielleicht verrätselte - Erwartung, die am Schluss eingelöst ist. So entsteht eine einheitliche Bewertung oder Perspektivierung des ganzen Textes.

(2) Wenn man den Weg von Texten durch die Medien beobachtet, z.B. von den Äußerungen, die eine bestimmte Person auf einer Pressekonferenz gemacht hat, bis hin zu den einzelnen Zeitungstexten oder ins Radio und Fernsehen, sieht man, dass Idiome eine große Chance haben, von Text zu Text weitergegeben zu werden. Ein immer wieder zu beobachtendes Beispiel ist der Schritt in die richtige oder falsche Richtung.

Wenn ein Politiker die Garantie haben will, dass ein Statement von ihm durch alle Medien hindurch zitiert wird, dann verwende er ein entweder besonders auffälliges Idiom oder er wandle ein Idiom ein bisschen ab, wie im folgenden Beispiel der Schritt in die falsche Richtung, der auf dem Weg von der Agentur in die Zeitung als kümmerlicher Rest einer längeren Originaläußerung des Herrn Feigel übriggeblieben ist.

\section{Feigel kritisiert Bronfman}

Zürich/Bern. SDA. Sigi Feigel, Ehrenpräsident der Israelitischen Cultusgemeinde Zürich, kritisiert den Präsidenten des Jüdischen Weltkongresses (WJC), Edgar Bronfman, wegen dessen Fernbleiben an der Sitzung der Spezialfondsleitung am Montag. Die Nichtteilnahme sei ein ,Schritt in die falsche Richtung“".

(Basler Zeitung, 10.7.97)

Schritt in die falsche Richtung ist eine bewertende Formulierung, die ein explizit bewertendes Element (falsch) enthält. Doch werden von der Zeitung aus den Äußerungen Feigels nicht diejenigen Passagen weitergegeben, aus denen klar würde, was denn daran falsch sein soll und warum es falsch sei.

(c) Modifikationen

Kräuter und Rüben ist eine (von mir) beabsichtigte Abwandlung von Kraut und Rüben. Zur Abgrenzung von sonstigen Varianten hat sich für diese intentionalen Abwandlungen der Terminus „Modifikation“ eingebürgert. Wie vielfältig die Arten solcher Modifikationen sind, welche semantischen, textlinguistischen und pragmatischen Effekte sie haben können, in welchen Textsorten sie vorzugsweise vorkommen, das wurde z. B. in Burger/Buhofer/ Sialm (1982), Wotjak (1992), Sabban (1998), Balsliemke (2001) gezeigt. Zugleich gibt es jedoch auch starke Indizien dafür, dass Sprachspiele dieser Art - sofern sie in öffentlichen Texten vorkommen - an ihrem „dispersen“ Publikum vielfach vorbeigehen, dass hier eine krasse Diskrepanz zwischen 
Produktion und Rezeption besteht. ${ }^{5}$ Aufgrund vorliegender Untersuchungen (vgl. z. B. Hemmi 1994) ist zu bezweifeln, ob ein Text wie der folgende (aus einem Werbeprospekt für das Jugendkonto der Schweizer Post) seine - in diesem Fall jugendlichen - Adressaten wirklich ,erreicht" ${ }^{\text {“6 }}$ ob sie den Witz überhaupt nachvollziehen können, obwohl ja im Text sehr viel getan wird, um ihn verständlich zu machen.?

Gelbes Konto auf and you can make you on the socks

Mach dich ruhig auf die Socken. Mit deiner Postcard kannst du an 700000 Geldausgabeautomaten weitweit Bargeld beziehen (...) Ab 16 Jahren gibts die Postcard VISA (...) Ab 18 Jahren gibts die Postcard EUROCARD (...) Mit diesen beiden Karten bis du in der Schweiz und in der ganzen Welt gern gesehener Gast, Kunde oder Kundin.

Dies ist eine neue, bisher meines Wissens nicht klassifizierte Art von Modifikation, bei der ein deutsches Idiom (intentional) in eine englische Version übersetzt wird, die es im Englischen als solche gar nicht gibt. ${ }^{8}$ Die Bedeutung des Idioms wird dabei nicht tangiert, nur die englische Form dient als Attraktion.

Textbezogene Analysen - ebenso wie Befragungen von Vpn. - zeigen auch ein hohes $\mathrm{Maß}$ an Variabilität vieler Idiome. Die Wörterbucheinträge täuschen da häufig ein einheitliches Bild vor, das es in der Sprachwirklichkeit nicht gibt. Wie die kodifizierten Formen der Phraseologismen angesichts der empirisch realen Variabilität zu beurteilen sind, das ist eine noch offene Forschungsfrage.

${ }^{5}$ Eine kaum mehr zu überbietende Modifikationspraxis findet sich bei Karl Kraus. Kraus' Umgang mit Phraseologie ist jetzt vorzüglich dokumentiert in Welzig (Hg., 1999), wo zu jedem besprochenen Idiom auch die von Kraus kreierten Modifikationen verzeichnet sind. Kraus konnte mit einem sprachsensiblen intellektuellen Publikum rechnen, das empfänglich war für die von ihm intendierte „Trockenlegung des weiten Phrasensumpfes" (Die Fackel Nr. 1, 1899, S. 2).

${ }^{6}$ Zur altersspezifischen Phraseologiekompetenz vgl. Häcki Buhofer/Burger (1994) und Häcki Buhofer (2003).

${ }^{7}$ Hinzu kommt, dass nach verschiedenen empirischen Studien die jüngere Generation eine deutlich geringere Phraseologie-Kompetenz hat als die älteren Generationen, vgl. z. B. Häcki Buhofer/Burger (1994), Häcki Buhofer (2003).

${ }^{8}$ Der Prospekt enthält noch weitere, abenteuerliche „Übersetzungen“. Sogar ein Helvetismus (ein Büro aufmachen ,etwas sehr umständlich in Angriff nehmen') wird verenglischt:

Gelbes Konto auf and you make no office up.

Ein Büro aufmachen kannst du woanders. Mit yellownet erledigst du deine Zahlungen bequem und kostenlos von zu Hause aus. (...)

Hier findet allerdings zugleich eine semantische „Remotivierung "statt, insofern durch das lokale Adverb woanders die wörtliche Bedeutung des Phraseologismus evoziert wird. 


\subsection{Phraseologie und Metaphernforschung}

Die phraseologische Forschung zu semantischen Aspekten, insbesondere zur Idiomatisierung, hat von außen, von der sog. kognitiven Metapherntheorie, wichtige Anstöße erhalten. Es scheint mir aber, nach anfänglicher Euphorie, im Moment unklar, in welche Richtung sich diese Art von Forschung entwickeln wird. Die kognitive Metapherntheorie (vgl. etwa Lakoff/Johnson 1980) basiert auf der Annahme, dass die menschliche Kognition metaphorisch strukturiert sei, und dies, wie Lakoff und Johnson (1999) für ihr Konzept in Anspruch nehmen, in einem auch philosophisch relevanten Sinn. Die Sprache ist dabei nicht mehr und nicht weniger als eine Oberflächenrealisierung der kognitiven Strukturen, unter anderen Realisierungsformen wie etwa der Gestik. Beispiele für solche metaphorischen Modelle sind etwa AFFECTION IS WARMTH (They greeted me warmly), KNOWING IS SEEING (I see what you mean) (Lakoff/Johnson 1999, S. 50 und S. 53 f.).

Das hat zunächst nicht direkt mit Phraseologie zu tun, doch ziehen die Vertreter der Theorie von allem Anfang an auch Idiome ${ }^{9}$ unter anderen konventionellen Metaphern als Belege für die Existenz eines solchen kognitiven Apparats bei und behaupten damit explizit, dass solche Idiome nicht "tot", sondern „motivierbar" und damit semantisch „lebendig" sind.

Andererseits wurde innerhalb der Phraseologie die Metapher immer schon als ein zentrales Phraseologisierungsverfahren angesehen und untersucht, z. B. die mit den Körperteilen verbundenen Metaphern (sog. Somatismen wie etw. ins Auge fassen, jmdm. auf die Zehen treten). Durch die kognitive Metapherntheorie, besonders durch die starke Körperzentriertheit der Theorie, haben nun die phraseologischen Forschungen einen theoretischen Hintergrund bekommen, der Übereinstimmungen und Unterschiede der Idiomatik verschiedener Sprachen besser zu verstehen erlaubt. Viele Idiome sind aus dieser Perspektive nicht mehr vereinzelte, nur idiosynkratische Erscheinungen, sondern sie sind motivierbar aus systematischen, eventuell übereinzelsprachlichen metaphorischen Prozessen.

Eine Tagung in Ascona 2001 (vgl. Burger/Häcki Buhofer/Gréciano [Hrsg.] 2003) hat gezeigt, wie attraktiv diese Perspektive gerade für sprachund kulturvergleichende phraseologische Fragestellungen ist. ${ }^{10}$

Auf der anderen Seite ist es fraglich, ob sich Idiome - und nur um diese geht es mir hier - tatsächlich in so extensivem Maße dazu eignen, die Existenz einer metaphorischen Struktur der Kognition zu belegen. Ich möchte

${ }^{9}$ Ebenso wichtig sind in der kognitiven Metapherntheorie Verbindungen wie ein Ereignis trat ein, ins Leben treten, aus dem Leben scheiden, jds. Zeit ist gekommen (vgl. Baldauf 1997, S. 131, S. 148), die man als teilidiomatisch auffassen könnte - falls man die Verben eintreten, treten, scheiden, kommen in diesen Verbindungen noch als metaphorisch auffassen will.

10 Vgl. auch Iñesta Mena/Pamies Bertrán (2002), die zahlreiche ide. und einige nichtide. Sprachen vergleichen. 
nur stichwortartig ein paar Vorbehalte nennen, die aus phraseologischer Sicht gemacht werden müssen:

(1) Die Frage ist, inwieweit die Idiome in einem synchronen Sinn, auf die heutigen Sprecher bezogen, als Metaphern aktiv sind. Man schaue sich nur die vielen Idiome mit der Komponente Hand an. Bei einem Teil kann man die Motivation nachvollziehen, bei anderen aber keineswegs (z. B. jmd. an die Hand gehen, jmdn. an der Hand haben, letzte Hand an etwas legen ...). Zweifellos lassen sich die semantischen Übertragungsprozesse genetischhistorisch rekonstruieren, aber für die heutigen Sprecher sind sie vielfach kaum mehr aktiv. Hier wird u. U. Kraut und Rüben neben- und durcheinander aufgeführt, um eine möglichst weiträumige Geltung der metaphorischen Konzepte zu sichern.

(2) Es ist nicht damit getan, Idiome einem bestimmten metaphorischen Schema zuzuordnen. Es muss im Einzelfall auch historisch überprüft werden, wie sich Metaphernfelder unter konkreten historischen Bedingungen entwickeln und welchen Status sie heute haben. Auch dort, wo ein Idiom offensichtlich in ein metaphorisches Konzept hineinpasst - wie die Idiome, die das Kranksein des Menschen auf abstraktere Sachverhalte übertragen - ist es oft nicht der universale kognitive Hintergrund, der für die aktuelle Lebendigkeit des Idioms verantwortlich ist, sondern viel konkretere und kleinräumigere metaphorische Felder. Wenn gegenwärtig das Idiom eine bittere Pille für jmdn. sein (,für jmdn. sehr unangenehm, sehr schwer hinzunehmen sein' Duden 11) Hochkonjunktur hat, so ist das zeitgeschichtlich bedingt. Bittere Pille passt in einen gegenwärtig äußerst aktuellen, mit verschiedenartigen Bildspendern gespickten Diskurs, der in allen Medien geführt wird und der aus verschiedenen Bildspendern gespeist wird: Das Gesundheitswesen ist KRANK, ist ein Patient, der PIllen SCHLUCKT. Die wirklichen Patienten sind nicht nur buchstäblich krank und schlucken Pillen, sondern sie schlucken auch metaphorische bittere Pillen, nämlich die Prämien.

(3) Für die lexikographische Praxis und für den Fremdsprachenunterrricht sind die potentiellen Gemeinsamkeiten eine Sache, die andere ebenso wichtige sind eben die einzelsprachlichen z.T. starken, z. T. nur subtilen semantischen und pragmatischen Spezialisierungen, die den Gebrauchsbereich eines Idioms begrenzen (vgl. z. B. Cheon 1998).

Um nur ein Beispiel zu nehmen: Baldauf (1997) hat die Lakoffschen Metaphernmodelle an empirisch erhobenem deutschem Material darstellt. Dort findet sich das Modell EXISTENZ IST PRÄSENZ IN EINEM BEHÄLTER, und darin das Idiom einer Sache Tür und Tor öffnen (S. 131 im Textbeleg ,... die impulsiven Reaktionen Tür und Tor öffnen würden."). Dass das Idiom als Beleg für die Container-Metaphorik aufgefasst werden kann, ist wohl richtig. Aber vom linguistischen Gesichtspunkt her mindestens ebenso wichtig ist es zu sehen, dass das Idiom eine negative semantische Kom- 
ponente enthält: Die Sache, der Tür und Tor geöffnet werden, hat einen negativen Aspekt, wie das Beispiel in Duden 11 demonstriert: „Die neuen Wohnungsbaugesetze werden der Grundstücksspekulation Tür und Tor öffnen."

(4) Idiome erscheinen den Muttersprachlern häufig motiviert. Man hat den Eindruck, das „Bild“ zu verstehen. So haben wir als Deutschsprachige wohl keine Mühe, mit das Kind mit dem Bade ausschütten eine ganz konkrete Vorstellung zu verbinden. Das ist aber eher deswegen der Fall, weil wir das Idiom in dieser Bedeutung gelernt haben, als weil es $\mathrm{zu}$ irgendwelchen kognitiven Strukturen passt. Das sieht man sofort, wenn man Deutschlernern dasselbe Idiom vorlegt, sofern sie es noch nicht als solches gelernt haben.

\subsection{Phraseologie und Grammatik}

Eine seit den Anfängen der Phraseologieforschung umstrittene und bis heute nicht befriedigend behandelte Frage ist das Verhältnis von Phraseologie und Grammatik. Die Grundfrage ist: Wie kann eine Grammatik mit der Tatsache umgehen, dass phraseologische Phänomene einerseits in verschiedener Hinsicht vorgefertigte Einheiten sind, andererseits aber sich verhalten wie normale Verbindungen von Wörtern? Auf die beiden genannten Hauptgruppen bezogen: Sind phraseologische Phänomene vom Typ den Nagel auf den Kopf treffen in eine Grammatik integrierbar oder sind sie Versteinerungen, die sich grammatischer Modellierung widersetzen? Für die unauffälligen Phraseologismen stellt sich die Frage anders: Haben sie überhaupt grammatische Eigenschaften, die sie von nicht phraseologischen Konstruktionen unterscheiden? Hier ist nach wie vor Grundlagenarbeit zu leisten.

Bereits Rothkegel (1973) und Burger (1973) haben versucht, in Anlehnung an Arbeiten zum Englischen transformationelle Beschränkungen (wie es damals noch hieß) von deutschen Idiomen (also z. B. die vorhandene bzw. fehlende Passivierbarkeit) festzustellen und wenn möglich zu erklären. Das ist nicht recht gelungen, und dieser Forschungsstrang wurde nur hin und wieder aufgenommen (z. B. Keil 1997, S. 21 ff., Dobrovol'skij 2001). Schaut man sich heutige Grammatiken des Deutschen an, sieht man, dass man hier offenbar nicht sehr viel weiter gekommen ist.

Ich nehme als Beispiel die IDS-Grammatik von Zifonun, Hoffmann, Strecker und anderen (1997), die wohl als repräsentativ für den Stand der Diskussion innerhalb der germanistischen Grammatik gelten kann:

Phraseologie kommt an zahlreichen Stellen zur Sprache, teils implizit, teils explizit. Zunächst ist festzustellen, dass die Terminologie je nach Bearbeiter/in unterschiedlich ist. Im Register findet man „Phraseologismus“ und 
„Phraseolexem“. In Bd. 1 (S. 700) ist die Rede von „idiomatischen Ausdrücken“, „,idiomatischen Wendungen“ und „Phraseologismen“, wobei die Bezeichnungen offenbar synonym gemeint sind, in Bd. 2 (S. 1069) von "Phraseolexemen“, was wohl wiederum synonym ist zu den Bezeichnungen in Bd. 1.

Neben diesen Bezeichnungen für die phraseologischen Verbindungen werden immer wieder die Grundbegriffe „Festigkeit“, ,Formelhaftigkeit“, „Idiomatizität" verwendet, ohne dass sie aber irgendwo erklärt würden.

Im einzelnen kommt Phraseologie auf folgende Weisen zur Sprache:

1) Phraseologismen werden explizit thematisiert.

Z.B. werden unter „Einfache Prädikate mit idiomatischem Ausdruck“ „,idiomatische Wendungen oder Phraseologismen, die es auch in der Rolle von Prädikatsausdrücken gibt" (Bd. 1, S. 700), genannt.

„Phraseologismen haben auf den ersten Blick formal eine komplexe Struktur und scheinen nicht so ohne weiteres insgesamt ein Prädikat, insbesondere ein einfaches Prädikat, zu bilden. Es ist jedoch festzustellen, daß sie sich semantisch als eine Einheit darstellen, die sich dadurch auszeichnet, daß sie eine Bedeutung hat, die nicht voll aus den Bedeutungen ihrer Komponenten herzuleiten ist.

Ob in einem gegebenen Fall tatsächlich eine idiomatische Wendung vorliegt oder nicht, ist allerdings nicht immer so einfach festzustellen. Hier soll nur darauf hingewiesen werden, daß es im Deutschen solche Wendungen gibt, die als einfache Prädikatsausdrücke betrachtet werden können. Für Kriterien zur Bestimmung des idiomatischen Charakters kann auf die einschlägige Literatur verwiesen werden, so etwa Fleischer 1982 und Burger 1973. Anzumerken ist noch, daß idiomatische Prädikatsausdrücke oft hinsichtlich Person, Numerus, Tempus und Passivierbarkeit Beschränkungen unterliegen, die ihre Brauchbarkeit für die Zwecke der Prädikation ebenfalls einschränken.“ (Bd 1, S. 701)

Das ist die Formulierung eines Programms, das erst noch ausgeführt werden müsste. Der für die Grammatik entscheidende Punkt ist die Formulierung am Schluss: ,die ihre Brauchbarkeit für die Zwecke der Prädikation einschränken“. Wenn die Formulierung zutrifft, dann müssten die „Beschränkungen“ im einzelnen im Hinblick auf die „Zwecke der Prädikation“ behandelt werden.

In Bd. 2 werden unter „Klassen von Komplementen“ drei Arten „fester Verbindungen mit Verben und nominalen oder präpositionalen Bestandteilen“ unterschieden, bei denen die nominalen bzw. präpositionalen Bestandteile „auf den ersten Blick wie Komplemente aussehen, aus bestimmten Gründen aber nicht als Argumente des Prädikats gelten können" (S. 1066f.):

a) Nominalisierungs- und Funktionsverbgefüge (z. B. Abschied nehmen; zum Ausdruck bringen/kommen)

b) Phraseolexeme (z.B.Trübsal blasen) 
c) Prädikate mit „Objektinkorporation“ (z.B. wellenreiten, radfahren, Auto fahren, Ski fahren, Golf spielen, Klavier spielen, preisgeben, danksagen, Fuß fassen ...)

Es bleibt hier offen, ob damit der ganze Bereich derjenigen Phraseologismen abgedeckt ist, die ein Verb und ein Komplement enthalten, oder ob es sich nur um besonders prominente Typen handeln soll.

Völlig zu Recht wird gesagt, dass die drei Klassen in unterschiedlichem Grade verfestigt sind. Es bleibt jedoch ungeklärt, was unter „Festigkeit“ verstanden werden soll. Einerseits scheint Festigkeit als zentrales Kriterium der Phraseologizität zu gelten, andererseits steht „Festigkeit“ neben „Lexikalisierung" und „Idiomatizität", wobei „Lexikalisierung" und „Idiomatizität" ihrerseits auch nicht definiert werden. So wird dann gesagt, dass die Nominalisierungs- und Funktionsverbgefüge am wenigsten und die Prädikate mit Objektinkorporation am stärksten verfestigt seien. Letzteres zumindest scheint mir sehr zweifelhaft zu sein. Das Argument der Orthographie ( $\mathrm{Zu}-$ sammen- und Kleinschreibung) ist inzwischen (partiell) obsolet geworden (neu: Rad fahren statt radfahren), was man den Autoren der Grammatik natürlich nicht anlasten kann. Relevanter ist das Argument, dass „zum größten Teil Veränderung oder Konversenbildung nicht mehr möglich" sei (,Wellen reiten: *Wellen werden/wird geritten; Fuß fassen: *Er hat seinen Fuß gefaßt"). Das Argument relativiert sich allzu leicht: Einerseits ist es keineswegs der ,größte Teil“ der Gruppe, der diese Beschränkungen aufweist (die Verbindungen mit fahren oder spielen sind durchaus passivfähig und lassen „Veränderungen" $\mathrm{zu}$, was immer auch damit gemeint ist), andererseits findet man die gleichen Beschränkungen bei großen Teilen der „Phraseolexeme".

Dass man bei den drei Gruppen nicht von Argumenten des Prädikats sprechen kann - das ist ja der Aspekt, der ihnen gemeinsam sein soll -, wird bei den Ausführungen zu a) und c) einigermaßen klar, bei b) jedoch fehlt jegliche Begründung. Der Verweis auf die „Verfestigung“ im folgenden Satz, der wohl als Begründung gemeint ist, setzt eben gerade das voraus, was er erklären sollte: „Die nominalen Bestandteile von Phraseolexemen sind also unter formalen Valenzaspekten durchaus Komplemente; durch die Verfestigung und die Einbindung in ein komplexes Prädikat haben sie aber den Argumentcharakter verloren." (S. 1069) Man müsste eben wissen, was „Verfestigung" ist, wenn der Begriff argumentativ verwendet werden soll. ${ }^{11}$

Die Charakterisierung der "Phraseolexeme" ist sowohl in semantischer als syntaktischer Hinsicht unzureichend. Zur Semantik heißt es nur: „Charakte-

1 Die S. $1067 \mathrm{f}$. aufgezählten acht „Definitions- und Abgrenzungskriterien“ wie „feste Artikelselektion" oder ,aufgehobene Numerusopposition“ oder „syntaktische Anomalien" sind in keinerlei systematischen Zusammenhang gebracht, so dass unklar bleibt, inwiefern sie zur „Definition“ und „Abgrenzung“ (wovon eigentlich?) dienen können. 
ristisch für sie ist die Konkretheit in der Lexik, oft gekoppelt mit Bildlichkeit und Metaphorik.“ (S. 1069) Die Begriffe „Bildlichkeit" und „Metaphorik" werden offenbar als selbsterklärend aufgefasst - was sie keineswegs sind. (Auf die mit Konzepten wie „Bildlichkeit“ und „Bildhaftigkeit“ verbundenen Diskussionen kann ich hier nicht eingehen, das wäre ein Thema für sich.) In syntaktischer (und natürlich auch semantischer) Hinsicht aber ist irreführend, dass die Nennformen der Idiome z. T. unvollständig angegeben sind. Korrekt formuliert ist jemanden ins Bockshorn jagen, bei den Garaus machen, einen Korb geben fehlt aber jeweils das Komplement jemandem. Einer der in syntaktischer Hinsicht gerade zentralen Aspekte von Idiomen wird hier schon bei der Nennform vernachlässigt: dass sie nämlich "externe" Valenzen haben, die funktionieren wie bei nicht-phraseologischen Konstruktionen, und ,interne" Valenzen, deren Valenzstatus (Komplementstatus) eben gerade strittig ist. Die Nennform dem Fass den Boden ausschlagen ist in anderer Hinsicht problematisch: Der Ausdruck lässt nicht ein beliebiges Subjekt $\mathrm{zu}$ und auch eine semantische Einengung auf belebte Subjekte wäre nicht hinreichend. Tatsächlich wird er in der Regel mit dem Subjekt Das (schlägt ...) gebraucht, allenfalls mit anaphorisch oder kataphorisch verwendeten Nomina. Bei jmdn. laust der Affe würde man annehmen, dass das Akkusativobjekt mit beliebigen Nomina (unter der Bedingung, dass sie Menschen bezeichnen) besetzbar sei, obwohl es faktisch in der Regel mit dem Pronomen mich vorkommt. Um solche Restriktionen zu finden, genügt ein Blick ins Wörterbuch wie Duden 11.

Außer an diesen beiden Stellen werden bestimmte Typen von Phraseologismen im jeweiligen thematischen Zusammenhang der Grammatik behandelt. So finden sich unter „KM-Typen ${ }^{12}$ mit formelhaftem Ausdruck“ auch „,Routineformeln“, z.B. mit dem Kommentar „Routineformeln wie (1) Guten Morgen! [...] sind Höflichkeitsformen. Sie zu äußern ist höflicher als sie nicht zu äußern [...]“ (Bd. 1, S. 936) Hier wäre der Begriff „, formelhafter Ausdruck" in seiner Relation zu den anderen verwendeten Begriffen (Festigkeit usw.) erklärungsbedürftig.

Explizit werden auch „Paarformeln“ genannt und wiederum als ,formelhafte Ausdrücke" bezeichnet. Hier kommt der Aspekt zur Geltung, dass sich bei diesen Ausdrücken die „Stellungstendenzen für die Abfolge der Konjunkte“ besonders deutlich zeigen. ,Zahlreiche Zweierkoordinationen unterhalb der Satzebene sind zu Paarformeln geworden und damit in ihrer Reihenfolge fix. Beispiele: [...] fix und fertig, hieb- und stichfest [...]" (Bd. 3, S. 2444). Wenn bei diesem Typ die verfestigte Reihenfolge der Elemente das Kriterium für die „Formelhaftigkeit" der Ausdrücke abgibt, so ist dies offensichtlich ein

${ }^{12} \mathrm{KM}=$,kommunikative Minimaleinheit“. 
ganz anderer Gesichtspunkt als bei den Routineformeln, deren Formelhaftigkeit unter pragmatischer Perspektive charakterisiert wird.

2) Phraseologismen werden häufig als Beispiele für eine bestimmte grammatische Erscheinung gegeben. Dabei wird in aller Regel nicht gesagt, dass es sich um einen Phraseologismus handelt. Manchmal ist das unverfänglich, manchmal aber problematisch:

Wenn geläufige Werbeslogans (und andere „Geflügelte Worte“ aus der Belletristik) als Beispiele herangezogen werden, so dient das eher der Erheiterung als der Verwirrung, so wenn unter „Generische Interpretation bei Nominalphrasen ohne Artikel“ als Beispielsatz gegeben wird: „Katzen würden Whiskas kaufen." (Bd. 3, S. 2061)

Ein Problem ergibt sich aber bereits bei Beispielen der folgenden Art:

„AcI-Konstruktionen sind Propositionalausdrücke, die nur in Verbindung mit Prädikaten auftreten können, die auf der Basis von Wahrnehmungsverben oder der Verben lassen und machen gebildet werden:

(14) Ich fühle die Schmerzen kommen.

(15) Dein dummes Gesicht macht mich nur lachen.

(16) Thomas hört wieder einmal das Gras wachsen.

(17) Die Professorin läßt den Assistenten ein Buch schreiben.“ (Bd.1, S. 756)

(14), (15), (17) sind nicht-phraseologische Konstruktionen. In (16), dem einzigen Beispiel mit hören, handelt es sich um das Idiom das Gras wachsen hören, das nicht fett hervorgehobene Verb ist also eine Komponente des Phraseologismus. Man könnte - auch als Muttersprachler - daraus irrtümlich schließen, dass der AcI mit dem Verb hören nur in diesem Idiom vorkommt.

Wenn mehrere Beispiele für eine grammatische Erscheinung gegeben werden, von denen das Idiom nur eines ist, kann man immerhin annehmen, dass sich das Idiom nach Meinung der Autoren in diesem Fall genau so verhält wie die anderen, nicht-idiomatischen Konstruktionen. (Das sollte allerdings explizit gesagt werden.) Problematisch hingegen ist es, für ein Phänomen überhaupt nur ein phraseologisches Beispiel zu geben, z. B.:

„Selbstverständlich sind auch in der Funktion eines Akkusativkomplements gegenstandsfundierte Nebensätze möglich.

(36) Was Hänschen nicht lernt, lernt Hans nimmermehr.“ (Bd. 2, S. 1087)

Von Sprichwörtern weiß man, ebenso wie von Idiomen, dass sie - synchron betrachtet - ungrammatische Konstruktionen aufweisen können. Im Fall (36) wird vom Leser - implizit - verlangt, dass er das Sprichwort als Realisierung einer ,normalen“ syntaktischen Konstruktion auffasst. 
Nicht akzeptabel scheint mir der Gebrauch eines Idioms als Beispiel wie in folgendem Fall, der sich unter dem Titel „Prädikativkomplemente" (Bd. 2, S. $1105 \mathrm{ff}$.) findet:

Von den Prädikativkomplementen heißt es, dass sie ,eine formal uneinheitliche Kategorie“ bilden, "die sich aber semantisch recht gut als eine Klasse beschreiben läßt" (Bd. 2, S. 1105). Sie treten mit einer Kopula oder einem kopulaähnlichen Verb auf, wobei die Verben ,einen vergleichsweise geringen Beitrag zum Aufbau der Satzbedeutung" leisten (Bd. 2, S. 1106). Beim Subtyp der ,adverbialen“ Prädikative erscheint dann eine Gruppe „Final" mit den Beispielen

(62) Das ist doch alles für die Katz.

(63) Dies Konzert ist für Elise/zum Entspannen. [usw.]

Beispiel (62) suggeriert, dass für die Katz semantisch den gleichen Status hat wie für Elise in (63). Das ist aber keineswegs der Fall, da für die Katz sein ein Idiom ist mit der Bedeutung, vergeblich, nutzlos' (Duden 11), in dem die Präposition wie auch das Nomen nicht ,wörtlich“ interpretierbar sind. ${ }^{13}$

3) Konstruktionen, die in phraseologischen Verbindungen vorkommen, sonst aber nicht oder nur beschränkt.

Es findet sich z.B. die Aussage, dass Verberststellung außer bei mögen

„,vorwiegend in formelhaften Wendungen sowie Sentenzen und standardisierten Mustern möglich [sei], ferner in Heischesätzen mit Indefinitausdrücken, die dem Aufforderungs-Modus besonders nahestehen, $z$. T. auch nicht von ihm differenzierbar sind:

(10) Hol' ihn der Teufel!

(11) Sei $M$ eine beliebige Menge.

(12) Nehme mal einer das Buch vom Tisch [usw.]" (Bd.1, S. 665)

Man kann hier nur vermuten, dass (10) als Beispiel für den Fall „,formelhafte Wendung" angeführt wird.

Als weiterer Fall sei die Behandlung von Ausdrücken wie eines schönen Abends genannt. Der Ausdruck wird als adverbialer Genitiv kategorisiert, und dann heißt es weiter: Der adverbiale Genitiv

„wird nur - in idiomatisch verfestigter Weise - für Zeitangaben genutzt. Genitivphrasen wie schlechter Laune, guten Mutes werden als Verbgruppenadverbialia und Prädikative gebraucht:

(6) Schlechter Laune nahm er an dem Bankett teil.

(7) Endlich einmal war sie wieder guten Mutes.“ (Bd. 2, S. 997)

${ }^{13}$ Freilich kann man durch einen geeigneten Kontext das Idiom ,remotivieren“ und die wörtliche finale Bedeutung aktualisieren. So im folgenden Satz aus einem Zeitungstext über ein Katzenheim, das Katzen nach dem Tod ihrer Halter betreut: „Bedenkt man, dass eine Katze durchaus 20 Jahre alt werden kann, ist das Erbe buchstäblich für die Katz." (Tages-Anzeiger, Zürich, 21.2.2003) In diesem Fall handelt es sich jedoch um eine semantisch modifizierte Verwendung des Idioms. 
Die ,idiomatische Verfestigung" soll offenbar nur für die Zeitangaben gelten, nicht aber für schlechter Laune und guten Mutes. Bei schlechter Laune ist das zutreffend, da man auch guter/mieser [...] Laune sein kann und Laune auch außerhalb der Konstruktion in der gleichen Bedeutung verwendet werden kann. Guten Mutes hingegen ist ein charakteristischer Grenzfall: Zwar lässt sich guten durch frohen/leichten/... substituieren, doch bedeutet Mut innerhalb der Phrase nicht dasselbe wie in nicht-phraseologischer Verwendung (Duden 11 gibt zu guten/frohen/leichten u. ä. Mutes sein: die Bedeutungserläuterung ,zuversichtlich, fröhlich gestimmt sein'). Das alles müsste gesagt sein, damit man die Formulierung „Genitivphrasen wie (...)“ richtig interpretieren könnte.

\section{4) Ausnahmen von Regeln}

„Wird in einen Prädikatsausdruck ein Ausdruck aufgenommen, der in der Regel modifizierende Wirkung hat, so liegt meist, aber nicht immer auch eine Spezifizierung des Prädikats vor. Es gibt weitgehend idiomatisierte Ausnahmen:

(28) Thomas ist ein Schwein.

(28') Thomas ist ein armes Schwein.

(29) Die Ergebnisse waren gut.

(29') Die Ergebnisse waren ganz gut.

(30) Mein Vater war Soldat.

(30') Mein Vater war ein alter Soldat." (Bd. 1, S. 709)

Hier wird suggeriert, dass der jeweils zweite Satz die idiomatisierte Ausnahme darstellt. Doch scheinen mir die drei Fälle ganz unterschiedlich gelagert zu sein: ein armes Schwein ist sicherlich idiomatisiert im semantischen Sinn. Bei ganz gut kann ich keine Idiomatisierung erkennen ( $g a n z$ ist in dieser Bedeutung mit vielen Adjektiven kombinierbar). Bei ein alter Soldat hat Soldat seine übliche Bedeutung, alt hat die Bedeutung ,ehemalig, einstig' (Duden GW, ,alt" Punkt 8), die auch in Kombination mit anderen Nomina vorkommt (nach Duden GW: alte Kollegen/Bekannte/die alten Plätze wieder einnehmen). Davon abgesehen, ist $\left(30^{\prime}\right) \mathrm{m}$. E. gar kein Gegenbeispiel gegen die ,modifizierende Wirkung“.

Einen sehr speziellen Fall stellt der folgende Passus dar. ${ }^{14}$

Unter dem Titel „Transitivierungskonstruktionen mit Prädikativen“ (Bd. 2, S. $1114 \mathrm{ff}$.) werden Formulierungen wie die folgenden aufgeführt

,a) Pavarotti treibt das Publikum aus dem Saal.

b) Pavarotti singt das Publikum aus dem Saal."

${ }^{14}$ Den Hinweis auf diesen Problemfall verdanke ich einem Vortrag von Irma Hyvärinen (,Kausative Resultativkonstruktionen als Phraseoschablonen im Deutschen und im Finnischen") in Loccum (2002). 
Satz a) ist eine unauffällige Konstruktion, der ähnlich aussehende Satz b) hingegen weist die Auffälligkeit auf, dass das Verb singen mit einem semantisch unpassenden Akkusativkomplement verwendet wird (singen hat sonst allenfalls ein ,inneres Objekt" wie Arie). Die Transitivierungskonstruktion in b) ist nicht phraseologisch, aber dennoch eine „Ausnahme“ von der üblichen Argumentstruktur des Verbs singen. Solche Konstruktionen unterliegen einer Reihe von syntaktischen und semantischen Beschränkungen (z.B. Kompatibilität zwischen dem Prädikativkomplement und seiner Bezugskonstituente). In den weiteren Ausführungen wird der „Ausnahme“-Charakter aber wieder relativiert: „Die Transitivierungsstruktur ist aber doch insoweit produktiv, als sich hier regelrechte Reihen bilden, die teilweise der Idiomatisierung unterliegen." (ebd., S. 1115) Als Beispiel für eine solche Konstruktion mit Reihenbildung wird die folgende gegeben:

,A übertrifft B durch X-en“

$\begin{array}{llll}\text { A trinkt } & \text { B } & \text { unter den Tisch } \\ \text { an die Wand } \\ \text { spielt } & & \text { in Grund und Boden } \\ \text { singt } & & \\ \text { redet } & & \\ \text { diskutiert } & & \end{array}$

Unklar bleibt bei der Formulierung, was denn hier genau ,der Idiomatisierung unterliege“. Die durch die Spaltenanordnung suggerierte Kombinationsfreiheit ist offensichtlich nicht gegeben. Es gibt zwar (gemäß Duden 11) das Idiom jmdn. unter den Tisch trinken (,mehr Alkohol vertragen als jmd., mit dem man trinkt'), aber nicht: jmdn. an die Wand trinken, es gibt jmdn. an die Wand spielen (,1. jmds. Einfluss [durch geschicktes Vorgehen] ausschalten, 2. jmdn. [bes. einen Sportler, Schauspieler o. Ä.] durch gutes Spiel deutlich übertreffen', aber nicht $j m d n$. unter den Tisch spielen usw. Mit anderen Worten: Es gibt eine regelmäßige Konstruktion und eine davon abweichende, bestimmten Beschränkungen unterliegende „Ausnahme“. Die Ausnahme ihrerseits aber ist wieder ,produktiv“, allerdings nur oder vor allem im Rahmen von Idiomen. Wenn das so ist, dann wäre das ein äußerst interessanter Grenzfall von Grammatik und Phraseologie, der allem, was sonst in der Grammatik als ,idiomatisiert“ bezeichnet wird, zuwiderläuft. Aber um die Argumentation in diesem Fall zu entwirren, müsste das Verhältnis von nicht-phraseologischen und phraseologischen Konstruktionen thematisiert werden.

\section{5) Grammatische Kriterien als Erklärung für Phraseologisierung}

Ein Ansatz zu einer grammatischen Erklärung einer phraseologischen Erscheinung findet sich bei der Besprechung der ,satzartigen Realisierungen“ von Komplementen, und zwar von W-Sätzen, deren „Einleiteelement im allgemeinen in seiner syntaktischen Funktion mit der des Subjunktorsatzes im 
Obersatz übereinstimmt“. Ein Teil solcher Subjunktorsätze bezieht sich ,auf beliebige Gegenstände und [sie] haben dann generalisierende Funktion, was sie für allgemeingültige Aussagen wie Gesetze, Sprichwörter, Sentenzen u. ä. zu einer besonders geeigneten Ausdrucksform macht. [...] (2) Was der Bauer nicht kennt, frißt er nicht." (Bd. 2, S. 1077)

Dies ist ein erhellender Hinweis darauf, wie Grammatik und Phraseologie interagieren können. Dass sich bestimmte Konstruktionen für bestimmte phraseologische Erscheinungen besonders eignen, gilt sicherlich nicht nur für Sprichwörter oder die im gleichen Zusammenhang aufzuführenden Gemeinplätze (vom Typ Was man hat, das hat man) und weitere satzwertige Phraseologismen (vgl. Lüger 1999), sondern vermutlich auch für andere Konstruktionen (z. B. „Modellbildungen“).

Das Fazit dieser Beobachtungen zu einer ausgewählten Grammatik ist dieses: Es kann nicht bei einem punktuellen oder gar stillschweigenden Einbezug phraseologischer Phänomene in eine Grammatik bleiben, sondern es bedürfte eingehender theoretischer Reflexion - von Seiten der Phraseologie wie der Grammatik -, um das Verhältnis von Grammatik und Phraseologie genauer zu bestimmen. Für das Englische kann man z. B. auf Jackendoff (1997) verweisen, der einen Grammatik und Phraseologie integrierenden theoretischen Entwurf vorgelegt hat.

Mindestens sollte eine Grammatik folgende Aspekte berücksichtigen:

- Die syntaktischen Typen von Phraseologismen sind darzustellen. Das ist schon deshalb nötig, damit man weiß, welche Strukturen im Deutschen ,,anfällig" sind für Verfestigung, Idiomatisierung usw.

- Wenn Begriffe wie Formelhaftigkeit, Festigkeit, Idiomatizität - ohne die auch eine Grammatik wohl nicht auskommt - verwendet werden, müssen sie zumindest rudimentär definiert und in ihrem inneren Zusammenhang dargestellt werden.

- Daran anschließen sollte eine systematische Behandlung der Kriterien für Phraseologisierung und entsprechender morphosyntaktischer „Tests“ (vgl. Anm. 10).

- Das Verhältnis von „interner“ und „externer“Valenz bei Phraseologismen sollte erläutert werden.

- Wenn phraseologische Beispiele verwendet werden, sind sie als solche zu deklarieren und es muss gesagt werden, ob es sich um Wortverbindungen handelt, die sich unter dem betrachteten grammatischen Aspekt wie nichtphraseologische, „normale“ Verbindungen verhalten.

Phraseologie ganz aus der Grammatik zu entfernen, ist sicherlich keine Lösung. Das wäre mindestens ebenso abwegig, wie wenn man die Idiomatisierung von Komposita und alle nicht völlig produktiven Muster nicht mehr im Rahmen der Wortbildung behandeln würde. 


\section{Perspektiven der Forschung}

Außer den genannten Problembereichen möchte ich noch weitere Perspektiven für die phraseologische Forschung nennen:

Früher war in der Phraseologie metaphorisch von „Kern“ und „Peripherie“ die Rede, wobei die Idiome der Kern und alles andere die Peripherie waren. Ich habe hier hauptsächlich vom „Kern" gesprochen. Aber es zeichnet sich eine Verlagerung vom Kern auf die Peripherie ab, von den Erscheinungen mit hochgradiger Idiomatisierung auf die schwach idiomatischen Verbindungen hin. Dies insbesondere unter dem Eindruck korpuslinguistischer Untersuchungen, die das Miteinandervorkommen von Wörtern als ein graduelles Phänomen betrachten und auch Phänomene schwacher Festigkeit aufdecken können.

Im Kontext von Deutsch als Fremdsprache werden - wie schon Bally gesehen hat - gerade die Kollokationen besondere Beachtung finden müssen.

Eine weitere theoretische und empirische Herausforderung für künftige Forschung ist es, die Verlagerung von "Festigkeit" zu „Variabilität“ in den Griff zu bekommen. Dem programmatischen Titel der Mannheimer Tagung 2003 (,Wortverbindungen mehr oder weniger fest") muss eine theoretische Basis gegeben werden und es bedarf entsprechender empirischer Untersuchungen, die einerseits an Korpora, andererseits mit Befragungen von Informanten durchgeführt werden können.

Vorerst noch scheint mir der Begriff der „Festigkeit“ - vor allem wenn man ihn differenziert genug verwendet - stark genug, um den Objektbereich der Phraseologie nicht als ein Feld voller Rüben und Kraut erscheinen zu lassen. Aber vielleicht ist es nicht mehr gerechtfertigt, die Idiome sozusagen als die Kräuter und den Rest als die Rüben anzusehen.

\section{Literatur}

Baldauf, Christa (1997): Metapher und Kognition. Grundlagen einer neuen Theorie der Alltagsmetapher. Frankfurt a.M. (= Sprache in der Gesellschaft 24).

Bally, Charles (1909): Traité de stylistique française. 2 Bde. Heidelberg.

Balsliemke, Petra (2001): „Das sieht die Welt schon anders aus.“ Phraseologismen in der Anzeigenwerbung: Modifikation und Funktion in Text-Bild-Beziehungen. Baltmannsweiler (= Phraseologie und Parömiologie 7).

Burger, Harald, unter Mitarbeit von Harald Jaksche (1973): Idiomatik des Deutschen. Tübingen (= Germanistische Arbeitshefte 16).

Burger, Harald/Buhofer, Annelies/Sialm, Ambros (1982): Handbuch der Phraseologie. Berlin/New York.

Burger, Harald (2003): Phraseologie - Eine Einführung am Beispiel des Deutschen. 2. überarbeitete Auflage. Berlin ( $=$ Grundlagen der Germanistik 36 ).

Burger, Harald/Häcki Buhofer, Annelies/Gréciano, Gertrud (Hg.) (2003): Flut von Texten - Vielfalt der Kulturen: Ascona 2001 zur Methodologie und Kulturspezifik der Phraseologie. Baltmannsweiler (= Phraseologie und Parömiologie Bd. 14).

Cheon, Mi-Ae (1998): Zur Konzeption eines phraseologischen Wörterbuchs für den 
Fremdsprachler. Am Beispiel Deutsch-Koreanisch. Tübingen (= Lexicographica. Series maior 89 ).

Dobrovol'skij, Dmitrij (2001): Pragmatische Faktoren bei der syntaktischen Modifizierbarkeit von Idiomen. In: Liedtke, Frank/Hundsnurscher, Franz (Hg.): Pragmatische Syntax. Tübingen (= Beiträge zur Dialogforschung Bd. 23). S. 271-308.

$\mathrm{DW}=$ Deutsches Wörterbuch von J. Grimm und W. Grimm (1854-1960). 16 Bde. Leipzig. Duden GW = Duden Das große Wörterbuch der deutschen Sprache in 10 Bänden (1999). Mannheim.

Duden 11 = Duden Redewendungen. Wörterbuch der deutschen Idiomatik (2002). 2. Auflage. Mannheim.

Duden Oxford = Duden Oxford. Großwörterbuch Englisch (1999). 2. Auflage. Mannheim et al.

Fleischer, Wolfgang (1982, 2. Aufl. 1997): Phraseologie der deutschen Gegenwartssprache. Leipzig bzw. Tübingen.

Fleischer, Wolfgang (1997): Das Zusammenwirken von Wortbildung und Phraseologisierung in der Entwicklung des Wortschatzes. In: Wimmer, Rainer/Berens, Franz-Josef (Hg.): Wortbildung und Phraseologie. Tübingen (= Studien zur deutschen Sprache 9). S. 9-24.

Häcki Buhofer, Annelies/Burger, Harald (1994): Phraseologismen im Urteil von Sprecherinnen und Sprechern. In: Sandig, Barbara (Hg.): Europhras 92 - Tendenzen der Phraseologieforschung. Bochum (= Studien zur Phraseologie und Parömiologie 1). S. 1-33.

Häcki Buhofer, Annelies (2003): Psycholinguistik der lexikalischen Lebendigkeit: Phraseologismenkenntnis in verschiedenen Lebensaltern am Beispiel einiger schweizerdeutscher Phraseologismen. In: Häcki Buhofer, Annelies (Hg.): Spracherwerb und Lebensalter. Tübingen/Basel. S. 279-292.

Hemmi, Andrea (1994): „Es muß wirksam werben, wer nicht will verderben“. Kontrastive Analyse von Phraseologismen in Anzeigen-, Radio- und Fernsehwerbung. Bern (= Zürcher Germanistische Studien 41).

Iñesta Mena, Eva Maria/Pamies Bertrán, Antonio (2002): Fraseología y metáfora: aspectos tipológicos y cognitivos. Granada.

Jackendoff, Ray (1997): The Architecture of the Language Faculty. Cambridge Mass. (= Linguistic Inquiry Monographs 28).

Jaksche, Harald/Sialm, Ambros/Burger, Harald (Hg.) (1981): Reader zur sowjetischen Phraseologie. Berlin.

Keil, Martina (1997): Wort für Wort. Repräsentation und Verarbeitung verbaler Phraseologismen (Phraseo-Lex). Tübingen.

Lakoff, George/Johnson, Mark (1980): Metaphors we live by. Chicago.

Lakoff, George/Johnson, Mark (1999): Philosophy in the flesh - The embodied mind and its challenge to Western thought. New York.

Lüger, Heinz-Helmut (1999): Satzwertige Phraseologismen. Eine pragmalinguistische Untersuchung. Wien.

Rothkegel, Annely (1973): Feste Syntagmen. Grundlagen, Strukturbeschreibung und automatische Analyse. Tübingen (= Linguistische Arbeiten 6).

Sabban, Annette (1998): Okkasionelle Variationen sprachlicher Schematismen. Eine Analyse französischer und deutscher Presse- und Werbetexte. Tübingen.

Welzig, Werner (Hg.) (1999): Wörterbuch der Redensarten zu der von Karl Kraus 1899 bis 1936 herausgegebenen Zeitschrift „Die Fackel“. Wien.

Wimmer, Rainer/Berens, Franz-Josef (Hg.). Wortbildung und Phraseologie. Tübingen (= Studien zur deutschen Sprache 9). 
Wotjak, Barbara (1992): Verbale Phraseolexeme in System und Text. Tübingen.

Zifonun, Gisela/Hoffmann, Ludger/Strecker, Bruno und andere (1997): Grammatik der deutschen Sprache. 3 Bde. Berlin (= Schriften des Instituts für Deutsche Sprache 7.1-3).

Elektronisch verfügbare Bibliographien:

www. europhras.unizh.ch („Neue Publikationen“)

www.ims.uni-stuttgart.de/euralex/bibweb (Bibliographie von Euralex und Europhras) 